\title{
Enhanced MR imaging of the shoulder, and sternoclavicular and acromioclavicular joint arthritis in primary hemochromatosis
}

\author{
Farzin Farpour · San J. Phan · Joseph Burns • \\ Jamshid Tehranzadeh
}

Received: 11 August 2009 / Accepted: 20 September 2009 / Published online: 14 October 2009

(C) The Author(s) 2009. This article is published with open access at Springerlink.com

\begin{abstract}
Shoulder arthritis is a common feature of hemochromatosis. However, a combination of sternoclavicle (SC) and acromioclavicular (AC) joint involvement associated with shoulder is uncommonly seen. We describe the MR imaging findings of the shoulder and SC and AC joint in a patient with primary hemochromatosis. MRI of the left shoulder revealed inflammatory arthritis and $1-\mathrm{cm}$ erosion of the humeral head, enlargement of the anterior glenoid labrum and formation of glenoid labral ovoid mass due to iron deposit. MRI of the AC joint showed inflammation and erosion with joint fluid. Significant contrast enhancement was noted at the SC joint due to inflammation.
\end{abstract}

Keywords Shoulder $\cdot$ SC joint $\cdot$ AC joint $\cdot$ Arthropathy . Hemochromatosis $\cdot$ MR imaging

\section{Introduction}

Hemochromatosis was first described by Trousseau in 1865 and was given its current name in 1889 by von Recklinghausen [1].

Hereditary hemochromatosis is an autosomal recessive disorder associated with the HLA-A locus on the short arm of chromosome 6 [2]. In normal subjects, absorption of iron from the gut is inversely correlated with iron stores. In homozygous hereditary hemochromatosis, there is an increased absorption of iron, which is not regulated by iron stores. This leads to progressive iron accumulation, because there is no normal mechanism for excretion of excess iron once it has been absorbed into the body [2].

F. Farpour $(\bowtie) \cdot$ S. J. Phan · J. Burns $\cdot$ J. Tehranzadeh University of California, Irvine (UCI)/VA, Irvine, USA e-mail: drffarpour@yahoo.com
Arthropathy occurs in $50-80 \%$ of patients with hemochromatosis, affecting initially the small joints of the wrist and hand, and later the larger joints of the body such as the hips, knees, shoulders and, rarely, ankles and elbows. Shoulder arthropathy is relatively common in primary hemochromatosis with radiologic features resembling those of osteoarthritis or calcium pyrophosphate dihydrate (CPPD) crystal deposition disease [3].

MR imaging has been shown to be the most sensitive and specific imaging modality in the detection of iron deposition in the solid viscera and myocardium [3], due to the strong paramagnetic properties of the stored iron. However, there are only a few prior studies that explain the MR imaging findings in arthropathy related to hemochromatosis in the hip [3], knee joint [4], wrist [5] and ankle [6].

We report on an enhanced MR imaging study of the shoulder, and acromioclavicular (AC) and sternoclavicle (SC) joints in a patient with primary hemochromatosis. To our knowledge, this is the first case report in English literature of the shoulder with AC and SC joint arthropathy due to primary hemochromatosis on MR imaging.

\section{Case report}

This 72-year-old man presented with left shoulder and AC joint pain and swelling, which progressively got worse over the past several months. He also had difficulty in raising his arm above his head. Left shoulder pain over the past several months was associated with a palpable mass along the medial aspect of the clavicle. He was a patient with known primary hemochromatosis and was homozygous for the C282Y mutation. This genotype is consistent with hereditary hemochromatosis. 
Fig. 1 a Hemochromatosis of the shoulder. AP radiograph of the left shoulder shows linear chondrocalcinosis (linear calcification) (arrow) of the hyaline articular cartilage surface of the humeral head. There is small calcification at the acromioclavicular joint. b Hemochromatosis of the shoulder. AP view of the pelvic shows chondrocalcinosis of the symphysis of pubis and bilateral hip replacement. Vascular calcification is also noted
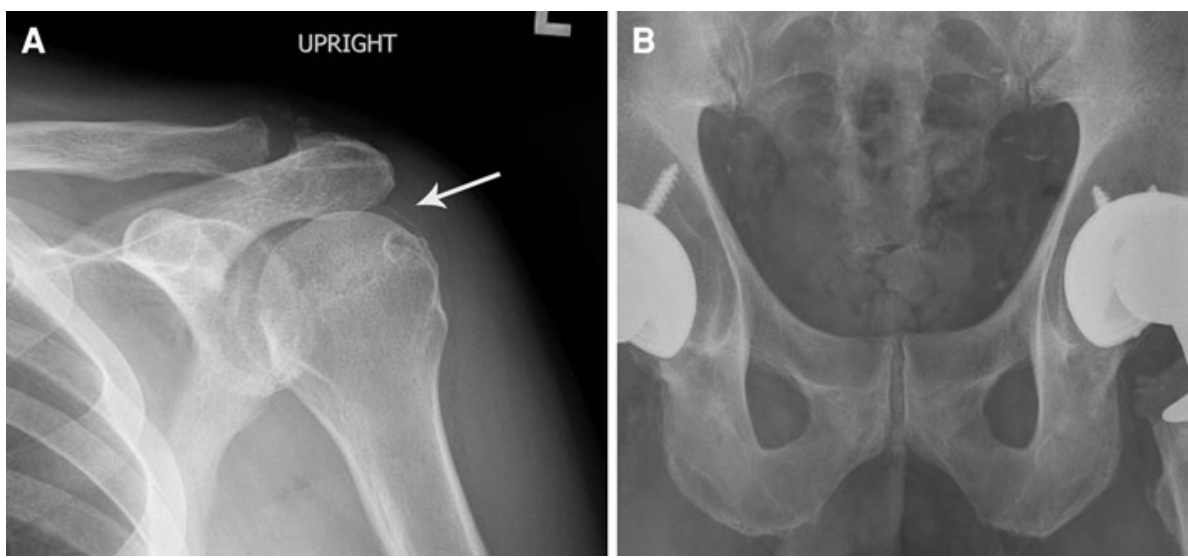

He had a past history of total hip replacement 13 years ago. Histologic sections of the articular surface of the femoral head revealed varying degrees of articular cartilage damage ranging from fibrillation to focal ulceration and changes in the subchondral bone, containing focal thinning and hemorrhagic changes in the base of the articular head. Mild intertrabecular fibrosis was also noted. Several areas showed a split in the articular cartilage at the border of the calcified and uncalcified cartilage. However, no refractile material (iron) was seen in the cartilage or in the subchondral bone.

On physical examination, he had swelling and pain in the medial aspect of the left clavicle and left AC and SC joint with associated neck stiffness.

Radiographs showed a focal erosion and linear chondrocalcinosis at the left humeral head and rotator cuff calcific tendinopathy Additional findings included degenerative changes at the AC joint and an old Hill-Sachs deformity.

MRI showed a 1-cm erosion at the humeral head, which enhanced with contrast. Additionally, there were subscapularis tendinosis, enlargement of anterior glenoid labrum, iron deposit causing blooming artifact at the tendon, joint and labrum, and formation of glenoid labral ovoid mass (GLOM). He also had erosion and inflammation with effusion at the AC joint. Synovial proliferation, inflammation and soft tissue prominence were also seen at the SC joint. Post-gadolinium images showed marked enhancement of the $\mathrm{AC}$ and SC joints, as well as the subacromion/subdeltoid bursa due to bursitis.

\section{Discussion}

Hemochromatosis is an autosomal recessive disease with a prevalence of about 4 cases per 1,000 in Europe and North America. One of every eight Americans is a heterozygous carrier. The heterozygous state is rarely symptomatic. Hemochromatosis is less common in black people of any ethnic origin [1].
Hemochromatosis is similar to CPPD crystal deposition disease/pseudogout. It produces radiographic changes similar to osteoarthritis, such as cartilage and joint narrowing, and chondrocalcinosis.

The development of arthropathy in hemochromatosis patients appears to be intimately related to the deposition of small amounts of iron or hemosiderin within the affected joints. Ferric salts promote the formation and deposition of intra-articular calcium pyrophosphate crystals by inhibiting the activity of synovial pyrophosphates and decreasing the clearance of intra-articular immune complexes, by inhibiting the activity of synovial reticuloendothelial cells. This often manifests as chondrocalcinosis on radiographs (Fig. 1a, b). These effects, reflecting systemic iron excess,

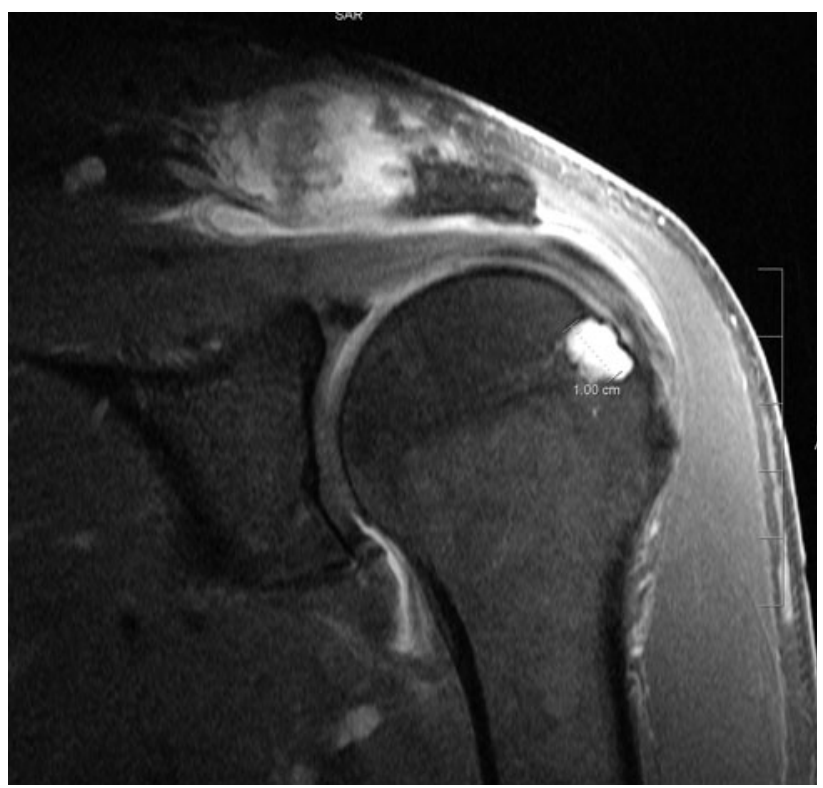

Fig. 2 Hemochromatosis of the shoulder. Coronal T1 fat-saturation post-contrast image (580/13) of the left shoulder shows a 1-cm erosion of the humeral head with contrast enhancement. Erosion and synovial enhancement are at the AC joint. There is enhancement of the subacromion subdeltoid bursa due to bursitis 


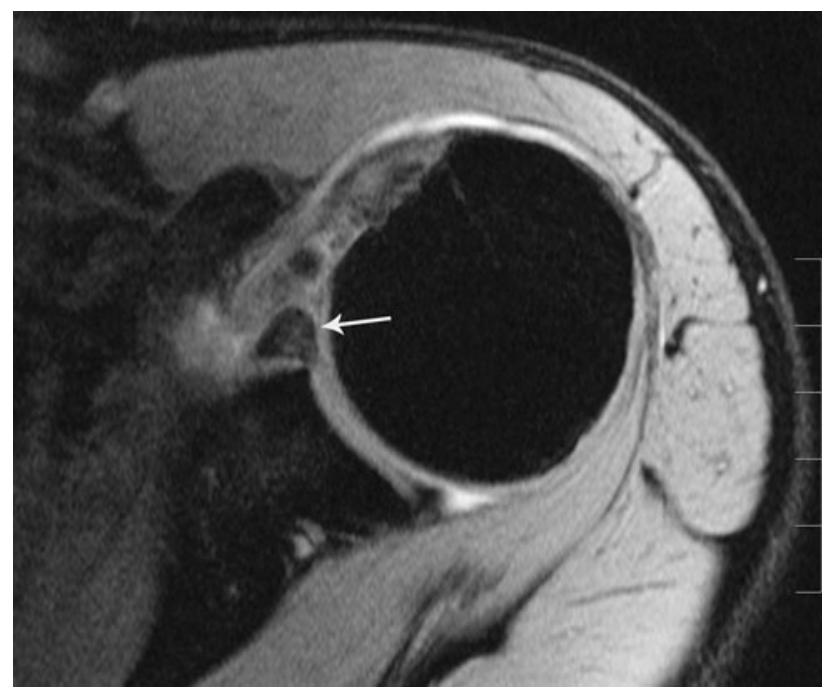

Fig. 3 Hemochromatosis of the shoulder. Axial T2 Medic image (gradient-echo sequences or GRE) (571/22) shows tendinosis of the subscapularis tendon and enlargement of the anterior glenoid labrum. Hemosiderin deposit causes blooming artifact at the tendon, joint and labrum, forming glenoid labral ovoid mass (GLOM) appearance

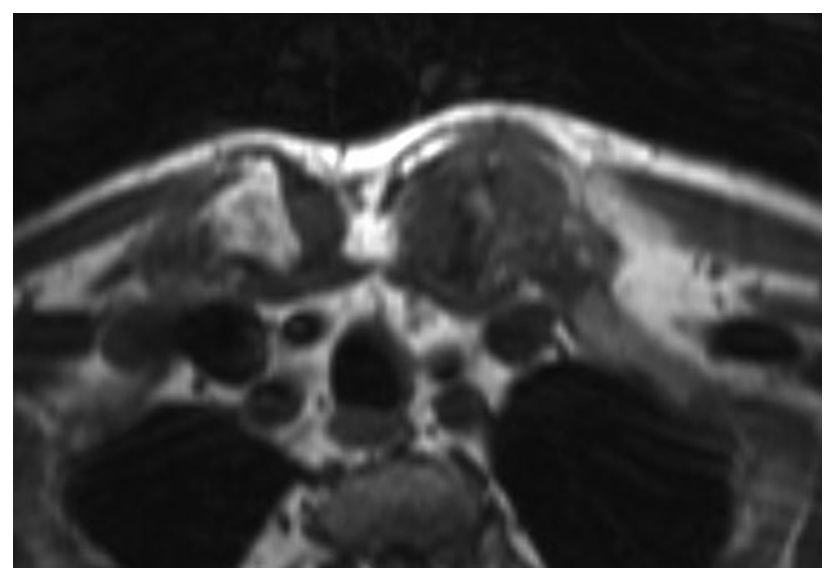

Fig. 4 Hemochromatosis of the sternoclavicular joint. Axial T1-weighted image (564/15) shows synovial proliferation and prominence of the sternoclavicular joint

appear to trigger the development of acute joint inflammation in the affected patients [4].

A pattern of arthritis involving the metacarpophalangeal (MCP) and proximal interphalangeal (PIP) joints of the second and third fingers suggests hemochromatosis [1] but the wrists, hips, knees and shoulders can also be involved [7]. However; its appearance can be indistinguishable from osteoarthritis, gout or pseudogout. Because iron overload disease underlies many cases of chondrocalcinosis, a radiographic finding of chondrocalcinosis should prompt evaluation for hemochromatosis [1].

Other radiologic findings include cystic joint erosion, hook-like osteophytes, joint space narrowing and sclerosis

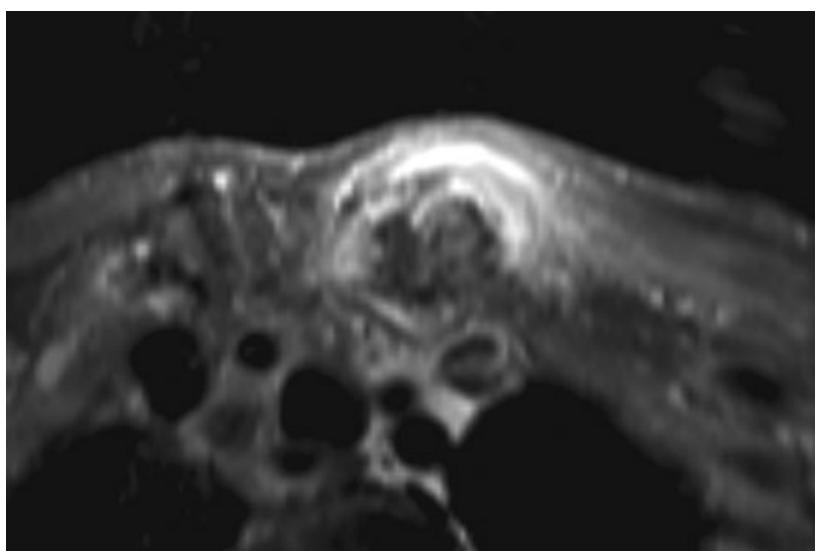

Fig. 5 Hemochromatosis of the sternoclavicular joint. Axial T1 with fat saturation post-contrast image (680/15) shows marked enhancement due to inflammation of the synovial tissue at the sternoclavicular joint

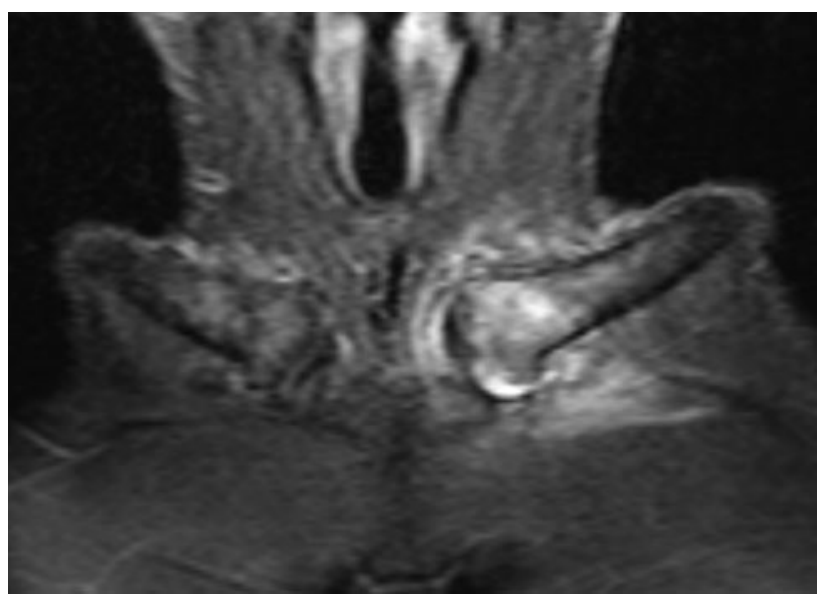

Fig. 6 Hemochromatosis of the sternoclavicular joint. Coronal STIR image (4930/55) shows marked increased signal at the left sternoclavicular joint with proximal clavicular bone marrow edema

[7]. Occasionally, joint cartilage is rust-colored, but this finding is not specific. Many people with joint replacement, particularly at younger ages, have hemochromatosis that is often unrecognized [1]. Our case also had bilateral hip joint involvement followed by total hip replacement at age 59 years (Fig. 1b).

Roan et al. [8] believed that GLOM can be caused by torn labral fragment, loose body, prominent middle glenohumeral ligament (MGHL) and dislocated biceps tendon.

Parmar et al. described a GLOM lesion as an interesting appearance of the anterior labral tear that had retracted superiorly. He also stated that GLOM represents a dark, rounded mass of tissue on proton density and T2-weighted images [3, 9]. However, our patient had enlargement of the anterior glenoid labrum (GLOM) due to hemochromatosis deposit (Fig. 3, arrow)

Huaux et al. [10] noted that the pattern of the hemochromatosis arthropathy is characterized mainly by articular 
chondrocalcinosis (ACC) and subchondral arthropathy. Jäger et al. [11] reported that $30 \%$ of patients with hemochromatosis have chondrocalcinosis and 50\% have degenerative osteoarthritis. Radiograph of the left shoulder in our patient showed calcific tendinopathy and linear chondrocalcinosis at the articular surface of the humeral head. AP view of the pelvic also showed chondrocalcinosis of the symphysis of pubis (Fig. 1a, b).

Schmid et al. reported that the MR imaging appearance of three ankle joints with primary hemochromatosis, containing extensive subchondral cyst formation, subchondral sclerosis, and joint space narrowing $[3,6]$. In our case MRI of shoulder shows a $1 \mathrm{~cm}$ erosion of humeral head (Fig. 2).

It is remarkable that the severity of osteoarthritis has not been found to correlate with the amount of intra-articular iron or total iron stores [3]. Moore et al. referred to susceptibility artifact due to iron deposition in the wrist joint [3, 5]. MR imaging has been used for detection and quantification of iron overload in the liver, spleen, and pancreas [3], as the strong paramagnetic properties of the stored iron cause a significant decrease in $\mathrm{T} 2$ relaxation times of the tissues. Iron accumulation in the synovium, cartilage or synovial fluid is considerably less pronounced than that of the solid viscera and is well below the threshold that can be reliably detected with MR imaging [3]. In a previous study, MR imaging failed to confirm the presence of intra-articular iron in the knee joint in ten patients with primary hemochromatosis [3]. However, in our patient, signal loss associated with iron deposit was diffuse and seen in the cartilage, synovial tissue and tendon on gradient sequences, which are considered to be the most susceptible to the paramagnetic properties of iron (Fig. 3) [3].

In conclusion, we described the MRI and radiographic findings of multiple joint involvement in a patient with primary hemochromatosis (Figs. 4, 5, 6).
Open Access This article is distributed under the terms of the Creative Commons Attribution Noncommercial License which permits any noncommercial use, distribution, and reproduction in any medium, provided the original author(s) and source are credited.

\section{References}

1. Felitti VJ (1999) Hemochromatosis: a common, rarely diagnosed disease. Permanent J 3(1):10-22

2. Baur LHB (2009) Patient screening for cardiac hemochromatosis, echocardiography or MRI? Int J Cardiovasc Imaging 25:249-250. doi:10.1007/s10554-008-9400-7

3. Papakonstantinou O, Mohana-Borges AVR, Campell L, Trudell D, Haghighi P, Resnick D (2005) Hip arthropathy in a patient with primary hemochromatosis: MR imaging findings with pathologic correlation. Skeletal Radiol 34:180-184. doi:10.1007/s00256004-0813-7

4. Eustace S, Buff B, McCarthy C, MacMathuana P, Gilligan P, Ennis JT (1994) Magnetic resonance imaging of hemochromatosis arthropathy. Skeletal Radiol 23:547-549

5. Moore EA, Vennart W, Jacoby RK et al (1993) Magnetic resonance manifestations of idiopathic hemochromatosis in the wrist. Br J Rheumatol 32:917-922

6. Schmid H, Struppler C, Braun GS, Kellner W, Kellner H (2003) Ankle and hindfoot arthropathy in hereditary hemochromatosis. J Rheumatol 30:196-199

7. Wheeler CJ, Kowdley KV (2006) Hereditary hemochromatosis a review of the genetics, mechanism, diagnosis, and treatment of iron overload. $132: 10-16$ (ISSN 1559-1190)

8. Roan KR et al (2004) Essentials of shoulder MR arthrography: a practical guide for the general radiologist. Clin Radiol 59:327-334

9. Parmar H, Jhankaria B, Maheshwari M, Singrakhia M, Shanbag S, Chawla A, Deshpande S (2002) Magnetic resonance arthrography in recurrent anterior shoulder instability as compared to arthroscopy: a prospective comparative study. J Postgrad Med 48:270-274

10. Huaux JP, Geubel A, Koch MC, Malghem J, Maldague B, Devogelaer JP, Nagant De Dexchaisenes C (1986) The arthritis of hemochromatosis. A review of 25 cases with special reference to chondrocalcinosis, and a comparison with patients with primary hyperparathyroidism and controls. Clin Rheumatol 5(3):317-324

11. Jäger HJ, Mehring U-M, Götz GF, Neise M, Erlemann R, Kapp HJ, Mathias KD (1997) Radiological features of the visceral and skeletal involvement of hemochromatosis. Eur Radiol 7(8):1199_ 1206 